\title{
Protée
}

\section{Le temps et la construction du sens}

\section{Bilha Blum}

Volume 27, numéro 1, 1999

La Mort de Molière et des autres

URI : https://id.erudit.org/iderudit/030536ar

DOI : https://doi.org/10.7202/030536ar

Aller au sommaire du numéro

Éditeur(s)

Département des arts et lettres - Université du Québec à Chicoutimi

ISSN

0300-3523 (imprimé)

1708-2307 (numérique)

Découvrir la revue

Citer cet article

Blum, B. (1999). Le temps et la construction du sens. Protée, 27(1), 29-32.

https://doi.org/10.7202/030536ar

\section{Résumé de l'article}

Cette analyse de La Mort de Molière fait l'examen de la juxtaposition de deux conceptions du temps, l'une conventionnelle et extratextuelle, et l'autre non conventionnelle mais présente dans le texte. Cette perturbation constante de la continuité du temps et de son irréversibilité, présente dans la vidéo, entraîne l'établissement d'un ensemble complètement différent de traits attribués à cette notion. La confrontation qui s'ensuit entre les deux conceptions du temps reflète la confrontation entre monde réel et monde fictionnel et, par conséquent, elle peut être utilisée comme principe interprétatif pour décoder cette oeuvre. 


\section{LE TEMPS ET LA CONSTRUCTION DU SENS}

Traduit de I'anglais par Jean-Pierre Vidal

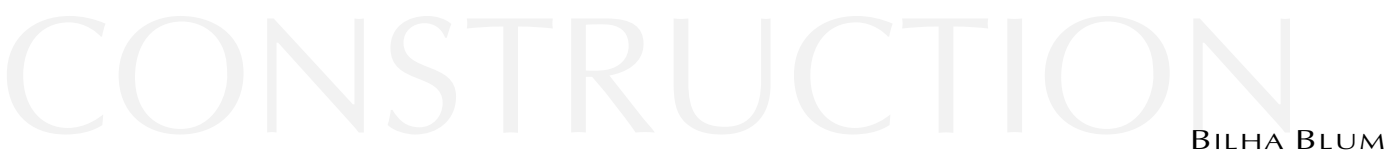

Il y a le moment pour tout, et un temps pour tout faire sous le ciel:

Un temps pour enfanter, et un temps pour mourir; un temps pour planter, et un temps pour arracher le plant. Un temps pour tuer, et un temps pour guérir [...] (L'Ecclésiaste, 3:1)

Lu de façon non orthodoxe, le texte de L'Ecclésiaste ${ }^{1}$ semble attribuer au temps le pouvoir rare et presque divin de régenter la vie, la mort et toute activité humaine de quelque importance. La nature temporelle de la vie et la conscience de la mort - qui contraste violemment avec le sens de l'infini attaché au temps sont en effet des certitudes profondément ancrées et difficilement contestables. Des termes comme «irréversible», «inaltérable» et «inévitable», qui servent souvent à décrire à la fois la fin de la vie et le passage du temps, ne font que confirmer ce rapport symbiotique et épistémologique.

De plus, la vie et le temps ont toujours été conçus métaphoriquement à travers l'histoire comme "fuyant» de la même façon. Le courant dominant de la philosophie occidentale considère généralement les deux notions comme linéaires, unidirectionnelles et continues, et, par ailleurs, existant à un niveau métaphysique situé bien au-delà de toute pénétration humaine. Mais la différence entre les deux tient au fait que si la vie se termine naturellement par la mort, le temps, lui, n'a pas de fin et, comme le prétend la modernité, amène un progrès constant. Par opposition à la vie, le mouvement perpétuel du temps vers le futur est ainsi censé engendrer l'espoir et assurer une certaine amélioration. Depuis les Lumières, en particulier, le passage du temps a donc été considéré, par-dessus tout, comme un processus incessant d'évolution apportant constamment à l'humanité de nouveaux bienfaits et un bien-être sans cesse accru ${ }^{2}$.

La Mort de Molière remet en cause cette conception traditionnelle du temps; à travers cette remise en question et par ses affinités avec la mort, l'œuvre remet également en question la conception du cycle vie-mort tout entière. Cette obsession du temps est une caractéristique commune à toutes les œuvres de Wilson, au point même qu'il est désormais possible de dire qu'elles reflètent une 
véritable «esthétique du temps» qui appartient en propre à l'artiste. Et en effet, dans nombre de ses premières œuvres, la manipulation que Wilson fait subir au temps a toujours été considérée comme une de leurs composantes artistiques majeures. Au moyen de tableaux vivants ${ }^{3}$ immobiles, d'un rythme extrêmement lent et, entre autres choses, d'une durée inhabituelle, Wilson tentait ainsi d'établir une correspondance étroite entre le temps théâtral et le temps réel, en unifiant l'expérience de l'espace temporel que pouvaient avoir les acteurs et le public.

Mais, dans La Mort de Molière, il abandonne son attitude habituelle et adopte au contraire une approche différente du temps qui, comme il se doit, résulte en une nouvelle façon de concevoir le rapport entre le temps, la mort et la création artistique. Ce changement d'approche se marque principalement par une rupture constante de la continuité du temps, et en particulier de son irréversibilité, grâce à divers dispositifs artistiques à l'œuvre dans la vidéo. Les deux, la continuité et l'irréversibilité, étant des caractéristiques propres au passage du temps, leur rupture est à l'origine d'une construction textuelle complètement différente.

La confrontation, qui tout naturellement s'instaure entre la conception conventionnelle du temps que se fait le spectateur et celle que le texte infère, ne fait que refléter une confrontation parallèle, celle qui oppose le monde réel et le monde de la fiction. On peut donc partir de là pour décoder l'œuvre dans son ensemble: en analysant la vidéo essentiellement sous l'angle de la conception du temps qu'elle met en œuvre, nous serons amenés à découvrir un important dénominateur commun entre les caractéristiques du temps textuel ainsi conçu et l'un des thèmes principaux de l'œuvre: la mort de Molière, ou plutôt, en fait, la mort en général.

Dans cette œuvre, Wilson a recours à des techniques artistiques de divers types pour construire sa conception singulière du temps, l'une des plus évidentes consistant sans doute à faire alterner des scènes de la biographie de Molière et la déconstruction de la chronologie du récit. Cet article s'attachera à une technique propre au médium choisi par Wilson, nommément le vidéotexte. Cette technique se manifeste par l'usage récurrent de flashes d'images interpolés tout au long de la vidéo et fonctionnant comme un moyen de rompre sa continuité linéaire. Ces flashes sont caractérisés par une suite d'images (ou inserts) qui n'appartiennent pas à la séquence dans laquelle elles sont placées. Par exemple, l'image des chaises en feu qui apparaît au début de la vidéo appartient en fait à la séquence montrant des danses rituelles de mort qui prend place à la fin de l'œuvre.

En quatre occasions différentes, le développement linéaire de l'œuvre est ainsi coupé par une séquence d'images très rapide, ou de flashes, qui apparemment n'ont rien à voir avec la situation montrée à l'écran à ce moment-là. Chacune de ces quatre séquences est faite soit d'images qui ont déjà été montrées, soit surtout d'images qui reviendront plus tard dans la vidéo, intercalées dans la séquence. De cette façon, le mouvement linéaire conventionnel de la vidéo, parallèle au cours naturel du temps, du passé au futur en passant par le présent, se trouve complètement perturbé. Le spectateur est ainsi confronté à un temps qui n'est ni continu, puisque son écoulement naturel est perturbé, ni irréversible, puisqu'il opère une série de va-et-vient entre le passé et le futur.

L'effet qui en résulte est le sentiment croissant que le présent se trouve en fait construit progressivement par le vidéotexte: un présent éternel, continu, sans fin, un présent gelé. Le temps est perçu comme pratiquement immobile, le futur devenant passé et le passé, futur. Au plan de l'interprétation, un tel cadre évacue naturellement des sentiments comme l'espoir ou l'aspiration à un changement et à une certaine amélioration; tout cela est rendu futile par l'apparente disparition de tout futur de quelque consistance. Il y a peu de chances de progrès dans une existence matérielle où le mouvement général du temps semble avoir pris un tour circulaire plutôt que linéaire. Incapable en fin de compte de franchir les frontières du présent, le spectateur est projeté en arrière plutôt qu'en avant, vers le point même d'où il est parti. 
Mais avant d'aller plus loin, j'aimerais spécifier le contenu des quatre séquences d'images, leur ordre d'apparition dans chacune des sections et la place des séquences dans la vidéo, pour bien faire percevoir la manipulation du temps qu'opère Wilson.

\section{Séquence 1}

Contenu: Chaises en feu, manuscrits, fauteuil vide vu de dos. Localisation: Section 2 de la vidéo.

Action en cours: On place quelque chose dans la main de

Molière - [Insertion de la séquence] - il porte la chose à sa bouche.

Séquence 2

Contenu: Télescope, neige qui tombe, chaises en feu.

Localisation: Section 4 de la vidéo.

Action en cours: Deux religieuses, l'une marmonnant en silence, l'autre vue de dos - [Insertion de la séquence] - Elles continuent à faire la même chose.

Séquence 3

Contenu: Manuscrits, religieuses en position d'adoration, cendrier, chaises en feu.

Localisation: Entre les sections 6 et 7 de la vidéo.

Action en cours: Le médecin essaie d'ouvrir la cage de verre contenant le fauteuil - [Insertion de la séquence] - Le

fauteuil vide apparait dans le cadre, toujours à l'intérieur de la cage de verre. Le médecin sort de l'image en marchant.

Séquence 4

Contenu: Manuscrits, fauteuil vide sur carré blanc, cendrier.

Localisation: Dernière section de la vidéo.

Action en cours: Une religieuse guide le père de Molière vers

le mur du fond - [Insertion de la séquence] - On voit le père de Molière à l'arrière-plan et la religieuse, ayant accompli sa tâche, regagne sa place.

Comme la description ci-dessus le montre clairement, la rupture du mouvement linéaire de la vidéo, parallèle à l'écoulement linéaire du temps, s'opère principalement par le rapport entre le lieu d'insertion des images, soigneusement choisi, et le contenu de ces images. Du point de vue de sa localisation, chaque séquence fige littéralement l'action en cours en interrompant pour un moment sa progression, sans lui causer la moindre altération.
Après les flashes, l'action reprend son cours, apparemment comme si de rien n'était. Du point de vue du travail artistique lui-même, ces images se marquent comme une parenthèse, une sorte d'entracte au cours duquel on rappelle aux spectateurs des événements passés, ou bien leur présente des événements futurs. Mais du point de vue de leur réception, ces images interfèrent avec l'impression normale de progression qu'ont les spectateurs qui, en conséquence, se trouvent pris dans un présent emphatique et sans fin.

Quant au contenu de ces images, son sens est rendu évident de deux façons, liées l'une à l'autre. Premièrement, comme je l'ai déjà dit, chaque séquence comprend à la fois des images du passé et des images du futur, qui servent, respectivement, de rappel de ce qui s'est déjà passé ou de prémonition de ce qui va se produire. L'effet de ces images sur l'histoire linéaire racontée par la vidéo (nommément, l'histoire de la mort de Molière) est particulièrement signifiant. En effet, si le temps est ainsi conçu comme circulaire - ce qui nous pousse à croire que nous existons dans un présent continu et continuel -, est-ce que la vie se termine vraiment avec la mort? $\mathrm{Ne}$ sommes-nous pas plutôt conduits, par cette coïncidence du passé et du futur, à percevoir la vie et la mort comme coexistantes?

Cette même notion de simultanéité se trouve mise en évidence tout au long de la vidéo. Un des exemples les plus forts est le plan montrant Molière, dans les tout derniers instants de sa vie, gisant sur son lit de mort, tandis que quelqu'un qui semble une version plus jeune de lui-même occupe le fauteuil autrefois vide et maintenant placé devant lui, au tout premier plan (section 5). La mort qui conduisait au vide, semble-t-il, est ainsi remplacée par la jeunesse, mais elles sont cependant toutes deux placées au même niveau d'existence. La forme presque cyclique de la danse rituelle à laquelle se livrent les religieuses dans la section 10, à la toute fin de la vidéo et juste avant la mort de Molière, sert à illustrer encore la même idée: le temps, comme la vie, comme la mort, n'a ni commencement ni fin. 
La deuxième façon dont le sens est suggéré par le contenu des images concerne l'usage de la technique de la répétition, qui est elle aussi caractéristique de la démarche artistique de Wilson. Les scènes, les actions ou les mouvements répétés ont toujours joué un rôle extrêmement signifiant dans son œuvre. L'impression que donnent généralement ces répétitions est celle d'un long processus continu qui semble ne jamais vouloir finir, dans la mesure où le passé (sous la forme d'une scène, d'une action ou d'un mouvement) ne cesse de revenir avec chaque répétition, créant ainsi finalement une impression d'immobilité. Un effet semblable est créé dans La Mort de Molière, où les éléments répétés comprennent plusieurs des images contenues dans les séquences avec flashes. Leur réapparition à l'intérieur de la structure spécifique des séquences ne fait pas que renforcer chez le spectateur l'impression d'un perpétuel présent, comme le font toutes les répétitions; elle en fait également un leitmotiv, une sorte de devise dont l'influence ne fait que croître avec chaque répétition. Le rôle joué, dans le processus d'ensemble de la construction du sens, par le contenu réel des images répétées, s'en trouve renforcé d'autant.

Les images qui réapparaissent dans les différentes séquences avec flashes sont celles qui montrent la crémation des manuscrits ( 3 fois), le feu qui sort des chaises ( 3 fois) et les cendres dans les cendriers ( 2 fois), le tout en très gros plans. Les trois types d'images montrent clairement leur dénominateur thématique commun, puisqu'elles contiennent une suite d'actions cohérente qui fait apparaître leur parenté: le feu qui sort des chaises va brûler les pages des manuscrits dont les cendres seront, quant à elles, placées dans les cendriers. En d'autres mots, même si le lien entre les images n'existe que dans l'esprit du spectateur (la scène complète de la crémation ne sera montrée que bien plus tard), elles peuvent être prises toutes les trois comme des représentations des diverses étapes du processus de destruction d'une création artistique.

Comme l'histoire que raconte la vidéo concerne Molière (un artiste et supposément l'auteur des manuscrits brûlés) et en particulier sa mort, on peut naturellement conclure que l'intention de l'auteur, telle que son œuvre la manifeste, était d'établir une connexion, une triple correspondance entre les notions de temps, de vie (et de mort) et de création artistique (et de sa destruction). Cette même correspondance, établie au plan visuel par les séquences avec flashes, se trouve explicitement confirmée au plan verbal également, comme le montre clairement la section 9 intitulée "Manuscrits". Outre évidemment la citation de Marlowe: «I'll burn my books», l'ensemble du texte de la section, fait de mots et de phrases apparemment sans suite, est littéralement construit comme s'il était emprunté à des pages en partie carbonisées. Malgré la confusion produite par ces mots épars, la mort, la guerre et la destruction sont au nombre des rares thèmes encore reconnaissables qu'il contient. Le lien direct entre les plans verbal et visuel ainsi établi - et dans cette œuvre il s'agit là d'une convergence plutôt rare - souligne encore plus l'importance de ces questions pour la compréhension de l'œuvre.

En conclusion, je dirai que Wilson se sert de ces séquences avec flashes, de leur localisation dans la vidéo et de leur contenu, pour illustrer le thème central de son ouvre, c'est-à-dire la nature de la mort. Ces séquences servent à rendre concret le concept abstrait d'un temps circulaire et non linéaire. Elles servent aussi à établir un rapport étroit entre le cycle de la vie et de la mort d'une part, et le processus de la création artistique d'autre part. La circularité de l'écoulement du temps vaut ainsi aussi bien pour le cycle de la vie que pour la pérennité de la création artistique. La circularité peut être interprétée comme une forme d'éternité, ou même d'immortalité, et, dans ce cas, elle renverrait ici à la nature éternelle de l'art. Mais elle peut aussi impliquer l'immobilité et la stagnation. Tout dépend de l'attitude de chacun.

\section{Notes}

1. La Sainte Bible, texte en français sous la direction de l'École biblique de Jérusalem, Bruges, Desclée de Brouwer, 1964, p. 985.

2. Pour un survol complet du développement du concept de temps dans la pensée occidentale, $c f$. C. M. Sherover, The Human Experience of Time, New York, New York University Press, 1975.

3. En français dans le texte (N.D.T.). 